\title{
Indirect Transmission of Head Lice via Inanimate Objects
}

\author{
Deon V. Canyon ${ }^{*}$ and Rick Speare
}

Anton Breinl Centre for Public Health and Tropical Medicine, James Cook University, Townsville QLD 4811, Australia

\begin{abstract}
Whether people can become infected by head lice transferring from inanimate objects is a topic of controversy. This paper reviews the evidence available from experimental studies in controlled laboratory experiments and data from field studies. The weight of evidence appears to be against transmission from inanimate objects being significant, and the promotion of inanimate objects that play an epidemiologically important role in head lice dispersal is not supported by evidence. We conclude that the control of head lice should focus on the head, not on the environment. However, additional studies are needed to quantify transmission risk via inanimate objects and the probability that head lice eggs survive and hatch off-host.
\end{abstract}

Keywords: Pediculosis, pediculus, transmission, fomites, inanimate objects.

\section{INTRODUCTION}

An assessment of transmission risks associated with the spread of pediculosis is important because the prevalence of Pediculus capitis, the head louse, has increased throughout the 1900s [1]. In terms of an infectious disease, when eradication is difficult or impossible, the next best option is control. This means interrupting the transmission cycle to limit the spread of disease agents [2].

Pediculosis is best managed by the individual or the carer who must act quickly to address infestation and transmission. Decreased pediculicide susceptibility and reinfestation make this difficult. Since insecticide susceptibility may vary substantially at a neighborhood level [3], it is best dealt with by adjusting treatment regimes. Reinfestation may involve direct transmission from other people or indirect transmission from inanimate objects. Direct reinfestation, involving head-to-head transmission, is highly subject to social factors and is thus difficult to resolve. Indirect transmission via inanimate objects would be a more resolvable issue.

This paper focuses on head lice transmission via inanimate objects. We review the body of knowledge in support of and against this transmission mode. It should thus be of practical use to policy developers and any person dealing with a recurrent pediculosis.

\section{SUPPORT}

The transmission of head lice by way of inanimate objects is not a clear-cut topic and there is difficulty in estimating the importance of direct (person-to-person) vs indirect (inanimate object-to-head) transmission. Since 10$20 \%$ of the children in most primary schools in most countries have head lice [1], a recommendation to change head lice policy in schools and management practices in the home can have significant economic implications. Several head lice scholars have devoted their efforts to determine the

*Address correspondence to this author at the Anton Breinl Centre for Public Health and Tropical Medicine, James Cook University, Townsville QLD 4811, Australia; Tel: 0431907814; E-mail: deoncanyon@gmail.com mechanisms involved in $P$. capitis transmission so that control programs do not squander limited resources [4]. The irony in this is that most head lice research is unfunded or suffers limited funding because head lice do not vector diseases and are simply considered to be an undesirable pest.

\section{CONTROVERSY}

Head lice mythology is strong in developed market economies and there is an abundance of unconfirmed suppositions that have inspired studies from different countries [5-11]. Some of these studies are field-based and employ epidemiological methods while others are laboratory-based and involve experimentation under controlled conditions. Other opinion-based publications provide little in the way of evidence and yet they put forward energetic arguments in support of strongly held views. These scholars and many others speculate as to the principal mode of transmission with varying conclusions because there is still a lack of data, and evidence-based results from field and laboratory sources are often contradictory. While experimental researchers demonstrate that inanimate objects are potentially important, the body of epidemiological evidence concludes that direct transmission from head-tohead is the primary mechanism.

For many years, the most active proponents of head lice transmission via inanimate objects have been the dermatologists Craig N Burkhart and Craig G Burkhart from the USA, while the most active proponents of direct head lice transmission have been Rick Speare, Petra Buettner and Deon Canyon from James Cook University in Australia. Both sides recognize that transmission from head-to-head and from inanimate objects occurs, but they differ in their belief in the extent to which each takes place. The former are advocates of anecdotal and experimental evidence, but they do not acknowledge most of the epidemiological and experimental evidence base produced by the latter and term it 'dogma' [2]. The latter recognize the former and are careful to include their perspectives and evidence in publications [12-15]. 
This article now endeavours to make a careful assessment of evidence at hand because this is not a simple academic argument, but one which affects people with pediculosis and their families. The controversy manifests itself in significant practical and economic outcomes for the infected population. The advocates of inanimate objects state that "louse control measures should ... include ... laundering of everything within the infested individuals' bed or quarantining of such material for 10 days, thorough vacuuming of floors, carpets, upholstery, with a standard vacuum cleaner" [2]. Advocates of direct, head-to-head transmission maintain that these measures are unnecessary, overly burdensome and pose an economic barrier to the effective management of head lice in all socio-economic contexts. Clearly the first approach, that of controlling lice on inanimate objects, is extremely time-consuming and costly while the latter approach, that of focusing control efforts on the head, is not.

\section{EVIDENCE IN SUPPORT OF INANIMATE OBJECTS}

Different modes of indirect head lice transmission were proposed in 2000 by Burkhart and Burkhart who state that inanimate objects can include [4]:

- Dislodged lice on inanimate objects moving onto a new host.

- Dislodged hairs carrying lice to a new host.

- Wind blowing lice from one location to a new host.

- Static electricity from combing which expels lice into the air and onto a new host.

In a recent review of fomite transmission by Burkhart and Burkhart (2007), the only evidence-based results provided in support of fomite transmission originated from one experimental laboratory study [16]. This study investigated fomite transmission in a number of ways. Firstly, a vacuum cleaner was passed over lice positioned on combs, towels or fabric to see if they could be dislodged. This does not approximate any realistic situation so the results are not very relevant to a realistic discussion. Secondly, lice were placed on 15 strands of hair and were subjected to a hair drier for a full minute. This also fails to correlate with reality because people never blast a hair dryer onto one spot on the scalp for a full minute. Thirdly, the lice on the 15 strands of hair were combed. This is nothing new since we know that combing is effective [15]. Fourthly, the lice on the 15 strands of hair were stirred in water and were gently towelled which resulted in transfer to the towel. This experiment also does not replicate reality where lice have access to a scalp and will run down a hair shaft onto the scalp to escape from external interference. Fifthly, a band of infested hair was placed onto a person's wrist and covered with dark cloth. After five minutes, $20 \%$ of lice had transferred onto the cloth. Had this final experiment been performed using a person's head, it would have been relevant. Any head lice researcher knows that lice will never remain on an arm and will always seek to move upwards until they reach the head. Thus this entire published study, which forms the 'evidence-base' of the transmission via inanimate objects theory is little more than a flawed collection of ill-devised and counter-intuitive experiments.

\section{EVIDENCE AGAINST INANIMATE OBJECTS - SUR- VIVAL OFF-HOST}

There are other factors to consider that relate to head-toinanimate object transmission and inanimate object-to-head transmission. One factor of key importance is that transmission of this nature is subject to the capacity of a louse to survive off-host long enough to come into contact with a new host and still have the strength to climb on board. While body lice may live up to 10 days off a host at $15^{\circ} \mathrm{C}, 5$ days at $24{ }^{\circ} \mathrm{C}$ and 3 days at $30{ }^{\circ} \mathrm{C}$ [17], this is not true for head lice which quickly perish from starvation when removed from a host $[6,18]$. Head lice only survive 9-11 hours at $25-37^{\circ} \mathrm{C}$ in low humidity and $10-14$ hours in higher humidity, but can survive up to 44 hours at $15{ }^{\circ} \mathrm{C}$ in higher humidity $[6,18]$. Most head lice perish 40 hours post-blood meal if they do not get another blood meal [19]. Thus the advice from Burkhart and Burkhart to quarantine the living quarters of infected people for 10 days [2] is not based on current evidence.

How these survival data impact on transmission rates from one substrate to another, whether living or inanimate, have not yet been determined by experiment although death after 2 days will stop any theoretical transmission far short of a 10-day quarantine period. In addition, fully active lice usually do not leave the head without any reason. Lice leaving the head may be close to dying, and it can be assumed that a high fraction of lice found off-head are not capable of infesting a person or surviving for very long. Data are required to confirm this assertion.

Whether or not head lice survival rates on inanimate objects are low or not, it is possible that gravid females could lay eggs that could later hatch and infest other hosts. Questions arising from this are: Do head lice off the host lay eggs on inanimate objects in nature? If yes, what are the survival and hatch rates of these eggs? In vivo-reared lice eggs take 8.4 days (range 6-11) to hatch with a hatch rate of $76 \%$ when they are exposed to a human host at night and are placed in a $20^{\circ} \mathrm{C}$ rearing chamber during the day. However, when eggs are exposed to a human for 8 hours each night they take 15.2 days to hatch and only $58-59 \%$ hatch $[16,20]$. While there are no available data for survival rates of eggs in unfavorable off-host circumstances, one may assume that the egg hatch rates off-host in nature is low due to dehydration. However, additional experimental evidence is needed to clarify this point.

\section{EVIDENCE AGAINST INANIMATE OBJECTS - FIELD STUDIES}

There are very few well-researched, field studies on the transmission of head lice via inanimate objects. One such study, an epidemiological investigation on New York schoolchildren, examined the sharing of lockers and wall hooks for winter clothing and headwear as a risk factor for pediculosis [5]. An association was found, however a replication of this study in Pennsylvania schools found no association [5].

Head lice researchers from the Anton Breinl Centre for Public Health and Tropical Medicine (ABC) at James Cook University in Townsville (Australia) conducted a series of specifically designed field studies to investigate the truth 
behind commonly mentioned inanimate objects. Their major, evidence-based, transmission-related findings related to three studies on the presence of lice on school hats, school floors and pillow slips of infested school children.

\section{Articles of Clothing as Inanimate Objects}

Hats have always been considered high-risk items. The general public and even many head lice researchers are of the opinion that hats and other apparel worn by infested people harbour lice. For instance, it has been stated that "Fomite transmission is common with Pediculosis capitis. The source of transfer is often headwear, shared hats, brushes, combs, earphones, bedding, upholstered furniture, and rugs" [4]. The validity of this anecdotal assertion was tested in a study in which over 1000 hats in four Australian schools were investigated for lice [21]. No head lice were found in all these hats and over 5500 head lice were captured from the heads of surveyed students who were wearing the very same hats. Thus, the results were $100 \%$ conclusive and found that school hats do not sustain head lice transmission. In another unpublished study conducted by the authors in a different primary school, a further 1000 hats were examined for lice and a single louse was identified on a hat belonging to a student whose hair had just been treated one hour earlier. It was assumed that this was a louse not killed by the treatment and was exhibiting the flee response. These empirical data demonstrate that the odds of head lice transmission occurring via transmission by the hats of children with pediculosis is sufficiently low to be considered improbable and inconsequential.

In conclusion, the transmission of head lice via inanimate objects including articles of personal clothing is in fact a well-established myth. When considering why it is so entrenched, one can only speculate that it originated from older generations more familiar with body lice residing in host clothing.

\section{Floors and Floor Coverings as Inanimate Objects}

Another common recommendation by many misinformed companies, web sites and articles in the popular press is to treat and clean rugs, carpeting or floors when pediculosis is found. This recommendation is based on the unsubstantiated belief that head lice are deposited on these surfaces with some regularity and would survive in the environment of the home or school long enough for people to become infested from this environment. However, no data exist in the scientific literature to support this.

Transmission of head lice from floors to people makes little evolutionary or biological sense, and the lack of data to quantify this potential route of transmission means that recommendations have not been evidence-based. This idea was put to the test by $\mathrm{ABC}$ researchers who carefully vacuumed the floors of 118 primary school classrooms in Australia and searched the contents for any signs of head lice [22]. A median of only $1.6 \mathrm{~g}$ of vacuum debris was obtained from each classroom prior to evening cleaning by school janitors. No head lice, parts of head lice or head lice eggs were found in any of this debris, but all samples contained parts of insects or occasionally whole insects. Ants and parts thereof were the most commonly identified insects found. This was notable because $21 \%$ ( 466 out of 2,230 children) who spent every school day in these 118 classrooms were infested with lice. A rather staggering total of 14,033 lice were captured from these 466 infested students and a mean intensity of infestation of 30.1 lice per infested child was calculated. Of the infested students, $58 \%$ had less than 10 lice, $35 \%$ had $10-99$ lice, $8 \%$ had $100-499$ lice, and one 4 year-old female preschooler had 1,623 lice. One hundred and eight of the 118 classrooms (91.5\%) had at least one student with active pediculosis and an average of 130 lice per infested classroom was calculated. Thus, the idea that lice are inclined to drop of a person's head onto the floor and that subsequent transmission takes place from floors to people is a fictitious myth that has been well and truly debunked. The risk of acquiring head lice from the floors of school classrooms is zero and there is no benefit in any control or cleaning activities to remove head lice from floors.

\section{Bedding as a Source of Head Lice}

If head lice contaminate bedding, it would pose a reinfection threat to people who have just been combed or treated with pediculicides. It would also pose an infection threat if other uninfested people were sharing the bed.

To resolve the question of whether or not bedding becomes infested by head lice leaving their host, researchers at the $\mathrm{ABC}$ investigated the proportion of head lice populations found on pillow cases of people with head lice, and tested strategies to kill head lice on pillow cases [20]. To assess the occurrence of head lice on pillow cases, people with active pediculosis had their head lice collected and counted and the pillow case they had used the night before examined for head lice. To test strategies to kill head lice on pillow cases, live head lice were experimentally placed in miniature pillow cases, and the cases subjected to a hot wash, a cold wash, a hot clothes dryer, and hanging out to dry on an outdoor clothes line in the sun.

In this study, 48 participants were recruited who were harbouring 1,845 head lice. Of the 48 pillow cases investigated, two cases had a single live nymph present and a third had a single dehydrated nymph. Thus, the occurrence of overnight live lice transference to pillow cases was $4.2 \%$ and proportionately, $0.1 \%$ of the head lice population actively transferred onto a fomite. The second part of the study found that heat (hot wash and hot clothes dryer) killed head lice experimentally placed in pillow cases while cold wash and hanging pillow cases out to dry did not kill head lice.

This low level of pillow case infestation provides further good evidence that bedding is an incompetent passive vector to the overall picture of transmission. Pillow cases do pose a slight risk for transmission or reinfestation but that risk is low enough to be considered unimportant compared to other modes of transmission. While this mode of indirect transmission is more important if bedding is shared, changing and washing pillow cases in a situation where multiple infected and uninfected people are cohabiting will have minimal impact on transmission since such a small proportion of the head lice population transfers to bedding, and bedding is a hostile environment for head lice. The point is for those advising on the control of head lice to look at the effectiveness of recommendations. Hence, this paper commented "Pillowcases pose a risk for re-infection with 
head lice, but the risk is low, and changing pillowcases is a reasonable cost-efficient strategy to mimimize this risk." Unfortunately, this was the only ABC field study cited by Burkhart and Burkhart who claimed that it provided evidence in support of "...the transfer of lice to numerous objects including hats, upholstery, headphones, and pillowcases..." [2].

It is entirely plausible that that head lice transmission occurs via inanimate objects to a significant extent in certain circumstances, but little more than anecdotal evidence exists to confirm this [23]. For instance, one of the authors (DVC) was once travelling in remote Aboriginal communities in northern Australia and happened one night to sleep in a 'men's house' with five other men. The author slept on the left of the group with his head approximately two feet away from the next person's head and at no time was his head in contact with the next person's head. Nevertheless, the author found himself infested the following day. No other head-tohead contact can be recalled before or after this event, which could have resulted in transmission. Head lice transmission may thus take place over inanimate objects between people who share bedding if the distance is small. But, the magnitude of this needs to be confirmed through appropriate scientific study. Other studies have found that family size, overcrowding and sleeping in the same bed can promote transmission [24-28].

\section{Brushes as Sources of Head Lice}

Three epidemiologic studies have demonstrated that there is no association between head lice presence and the sharing of combs and brushes, and one found an association [10, 29, 30]. In the latter study, which took place in Canada, no lice were found on the brushes of 10 children with active pediculosis [30]. The body of evidence thus suggests that this mode of transmission is possible, but most likely of little consequence compared to other modes.

\section{RECOMMENDATIONS FOR FUTURE RESEARCH}

As discussed, advice abounds on what to wash and clean if you have a head lice infestation, but the evidence for these assertions is anecdotal or based on flawed laboratory studies. Very little, if any, in the way of supporting field evidence is presented to back up these assertions. At the heart of the matter are a number of questions about healthy active head lice:

- How often do healthy head lice become naturally dislodged from their host?

- What do head lice do when they find themselves offhost?

- Do head lice on inanimate objects constitute a significant transmission problem?

- Do head lice actively leave their hosts to wait on inanimate objects for a chance occurrence with another host?

- Do head lice intentionally traverse inanimate objects to cross from one host to another in response to olfactory stimuli emanating from potential hosts?

- $\quad$ Do female head lice lay eggs when they are off-host?

- Do these eggs survive and hatch?
A comprehensive awareness of the biology and ecology of $P$. capitis and the epidemiology of pediculosis leads to the conclusion that transmission via inanimate objects may occur, but it is unimportant compared to direct person-toperson transmission. The emergence of data on transmission factors over the past decade has definitively enabled evidence-based head lice researchers to state that direct person-to-person transmission is the major route. However, transmission is a product of several inter-related variables, and physical factors pale into insignificance when compared to social and behavioural factors. The real problem is not how head lice transmission occurs, but why it is occurring. Reinfestation is thus is of greater importance, and future studies should endeavour to reveal the reasons behind it.

\section{CONCLUSIONS}

The control of head lice should focus on the head, not on the environment. Head lice scholars should focus on how to limit reinfestation rather than providing erroneous control advice. Schools and other institutions wishing to be proactive should invest their resources into educational approaches if they wish to render their mass treatment campaigns more effective. The promotion of inanimate objects that play an epidemiologically important role in head lice dispersal is unsupported by the evidence-base, and any further support of this mode of transmission needs to be accompanied by new conclusive evidence from welldesigned laboratory and field studies.

\section{REFERENCES}

[1] Gratz NG. Human lice: their prevalence, control and resistance to insecticides: a review 1985-1997. Geneva: World Health Organization, Division of Control of Tropical Diseases, WHO Pesticide Evaluation Scheme, 1997.

[2] Burkhart CN, Burkhart CG. Fomite transmission in head lice. J Am Acad Dermatol 2007; 56: 1044-7.

[3] Canyon DV, Hii JLK. Insecticide susceptibility status of Aedes aegypti (Diptera: Culicidae) from Townsville. Aust J Entomol 1999; 38: 40-3.

[4] Burkhart CN, Burkhart CG. The route of head lice transmission needs enlightenment for proper epidemiologic evaluations. Int J Dermatol 2000; 39: 878-9.

[5] Juranek DD. Pediculus capitis in school children. In: Orkin M, Maibach H, Eds. Cutaneous infestations and insect bites. New York, NY: Dekker 1985; pp. 199-211.

[6] Nuttall GHF. The biology of Pediculus humanus. Parasitology 1917; 10: 180-5.

[7] Mellanby K. Natural population of the head-louse (Pediculus humanus capitis: Anoplura) on infected children in England. Parasitology 1943; 34: 180-3.

[8] Slonka G, McKinley T, McCroan J, et al. Epidemiology of an outbreak of head lice in Georgia. Am J Trop Med Hyg 1976; 25: 739-43.

[9] Sinniah B, Sinniah D, Rajeswari B. Epidemiology of Pediculus humanus capitis infestation in Malaysian school children. Am J Trop Med Hyg 1981; 30: 734-8.

[10] Mumcuoglu K, Miller J, Gofin R, et al. Head lice in Israeli children: parents' answers to an epidemiological questionnaire. Pub Health Rev 1990-1991; 18: 335-44.

[11] Ebomoyi EW. Pediculosis capitis among urban school children in Ilorin, Nigeria. J Nat Med Assoc 1994; 86: 861-4.

[12] Heukelbach J, Canyon DV, Speare R. The effect of natural products on head lice: In vitro tests and clinical evidence. J Ped Infect Dis 2007; 2: 67-76.

[13] Heukelbach J, Canyon DV, Oliveira FA, Muller R, Speare R. In vitro efficacy of over-the-counter botanical pediculicides against the head louse Pediculus humanus var capitis based on a stringent standard for mortality assessment. Med Vet Entomol 2008; 22: 264-72. 
[14] Canyon DV, Speare R. A comparison of botanical and synthetic substances commonly used to prevent head lice (Pediculus humanus var. capitis) infestation. Int J Dermatol 2007; 46: 422-6.

[15] Speare R, Canyon DV, Cahill C, Thomas G. Comparative efficacy of two nit combs in removing head lice (Pediculus humanus var. capitis) and their eggs. Int J Dermatol 2007; 46: 1275-8.

[16] Takano-Lee M, Edman JD, Mullens BA, Clark JM. Transmission potential of the human head louse, Pediculus capitis (Anoplura: Pediculidae). Int J Dermatol 2005; 44: 811-6.

[17] Busvine J. Pediculosis: biology of the parasites. In: Orkin M, Maibach H, Eds. Cutaneous infestations and insect bites. New York: Dekker 1985; pp. 163-74.

[18] Payot F. Contribution a l'etude du Phthirus pubis (Linne, Leach). Bull Soc Vaud Natl 1920; 53: 127-61.

[19] Takano-Lee M, Yoon KS, Edman JD, Mullens BA, Clark JM. In vivo and in vitro rearing of Pediculus humanus capitis (Anoplura: Pediculidae). J Med Entomol 2003; 40: 628-35.

[20] Speare R, Cahill C, Thomas G. Head lice on pillows, and strategies to make a small risk even less. Int J Dermatol 2003; 42: 626-9.

[21] Speare R, Buettner PG. Hard data needed on head lice transmission. Int J Dermatol 2000; 39: 877-8.
[22] Speare R, Thomas G, Cahill C. Head lice are not found on floors in primary school classrooms. Aust N Z J Public Health 2002; 26: 208-11.

[23] Burgess I. Head lice. Clin Evid 2005: 2044-9.

[24] Andrews JR, Tonkin SL. Scabies and pediculosis in Tokelau Island children in New Zealand. J R Soc Health 1989; 109: 199-203.

[25] Kwaku-Kpikpi JE. The incidence of the head louse (Pediculus humanus capitis) among pupils of two schools in Accra. Trans R Soc Trop Med Hyg 1982; 76: 378-8.

[26] Sinniah B, Chandra S, Ramphal L, Senan P. Pediculosis among rural school children in Kelang, Selangor, Malaysia and their susceptibility to malathion, carbaryl, perigen and kerosene. J R Soc Helth 1984; 104: 114-5, 118.

[27] Jinadu MK. Pediculus humanus capitis among primary school children in Ife-Ife, Nigeria. J R Soc Health 1985; 105: 25-7.

[28] Chunge R. A study of head lice among primary school children in Kenya. Trans Roy Soc Trop Med Hyg 1986; 80: 42-6.

[29] Chunge R, Scott F, Underwood J, Zavarella K. A review of the epidemiology, public health importance, treatment and control of head lice. Can J Public Health 1991; 82: 196-200.

[30] Chunge RN, Scott FE, Underwood JE, Zavarella KJ. A pilot study to investigate transmission of headlice. Can J Pub Health 1991; 82: 207-8.

(C) Canyon and Speare; Licensee Bentham Open.

This is an open access article licensed under the terms of the Creative Commons Attribution Non-Commercial License (http://creativecommons.org/licenses/by-nc/ 3.0/) which permits unrestricted, non-commercial use, distribution and reproduction in any medium, provided the work is properly cited. 